\title{
El derecho a
}

la protección de datos personales desde un análisis

histórico-doctrinal*/

The Right to the

Protection of Personal

Data from a

Doctrinal-Historical

Analysis

* Recibido: 12 de mayo de 2014. Aceptado: 11 de junio de 2014 .

Tla-Melaua, revista de Ciencias Sociales. Facultad de Derecho y Ciencias Sociales. Benemérita Universidad Autónoma de Puebla, México / isss: 1870-6916 / Nueva Época, Año 9, № 38, abril / septiembre 2015, pp. 58-70. 
RESUMEN

La circulación ilimitada de los datos personales, dado el desarrollo de nuevas tecnologías, hace que los titulares no siempre encuentren suficientes mecanismos de control en el momento en el que se produce una alteración, distorsión o distribución de los mismos. Tales transgresiones plantean nuevos retos a los ordenamientos jurídicos, en aras de propiciar tutela a los titulares, lo cual constituye uno de los fundamentos del derecho a la protección de datos personales. Los posicionamientos teóricos de algunos autores y el reconocimiento constitucional y normativo que este derecho ha tenido permiten lograr una aproximación a los rasgos que lo distinguen y al proceso evolutivo en el cual ha estado inmerso. El presente artículo se centra en dicho particular. Realiza un acercamiento histórico-doctrinal, tomando como fundamento las particularidades presentes en Latinoamérica.

PALABRAS CLAVE

Datos personales, derechos fundamentales.
A B S TRACT

The unlimited circulation of personal data, due to the development of new technologies, does not allow the owners to find sufficient control mechanisms in place when there is an alteration, distortion or distribution of said data.

These transgressions propose new challenges to the legal system in order to provide protection to the holder, which constitute one of the foundations to the right to the protection of personal data. The theoretical positioning of some authors, the constitutional recognition and normative framework that this law has had achieves an approximation to its distinguishing features and to the evolutionary process in which it has been immersed. This article focuses on this particular law with a historical and doctrinal approach taking as a foundation the particularities existing in Latin America.

KEYWORDS

Personal Data, Fundamental Rights.

\footnotetext{
* Profesora en la Universidad de las Tunas, República de Cuba. (zahira@ult.edu.cu)
} 
1. Introducción / 2. Un acercamiento histórico-doctrinal / 3. Valoraciones desde un ámbito normativo; particularidades en Latinoamérica / 4. Conclusiones

\section{INTRODUCCIÓN}

Para acercarse al estudio histórico-doctrinal de cualquier derecho con rango constitucional, sin duda hay que hacerlo desde la comprensión de lo que ha sido llamado, por diversos autores, como generaciones de derechos. Uno de ellos ha sido Aristeo García González, ${ }^{1}$ quien las cataloga como categorías determinadas de acuerdo con contextos específicos, sin sustituirse un derecho por otro, sino que implican el surgimiento de otros para dar respuesta a nuevas necesidades o para redefinir los existentes.

Con base en ello se identifican tres generaciones de derechos. La primera pertenece a la época burguesa del siglo XVIII, cuando se marcaron los derechos individuales de defensa de la persona. La segunda se originó con las luchas sociales del siglo XIX y hasta el siglo Xx, con la formulación de la Declaración Universal de los Derechos Humanos (1948). La tercera se ubica a partir de la segunda mitad del siglo xx; en ella se incluyen los derechos de solidaridad.

$\mathrm{Al}$ referirse al innegable proceso evolutivo que sufren los derechos, Pérez Luño afirma:

El cambio de generación es esencial y expresa por tanto que el papel de los derechos fundamentales, deja de ser el mero límite a la actuación estatal para transformarse en instrumentos jurídicos de control de su actividad positiva, que debe estar orientada a posibilitar la participación de los individuos y los grupos en el ejercicio del poder. Lo que trae como consecuencia la necesidad de incluir en el sistema de los derechos fundamentales no sólo a las libertades clásicas, sino también a otros como categorías accionables y no como meros postulados programáticos. ${ }^{2}$

Con el avance científico, la vida humana experimenta cambios. Por ello, los derechos, al estar inmersos siempre en un proceso dinámico y nutriéndose de las relaciones sociales en su ámbito cultural, han ido adecuando sus instituciones y su normatividad en defensa de la persona. Todo lo cual ha sido

\footnotetext{
${ }^{1}$ García González, Aristeo, "La protección de datos personales: Derecho fundamental del siglo xxi. Un estudio comparado", Boletín Mexicano de Derecho Comparado, vol. 40, núm. 120, México, unam, 2007, p. 746.

${ }_{2}^{2}$ Pérez Luño, Antonio Enrique, Derechos Humanos, Estado de Derecho y Constitución, Madrid, Centro de Estudios Constitucionales, 1991, p. 228.
} 
evidente en el proceso de evolución del derecho a la protección de datos personales. El análisis histórico-doctrinal del mismo constituye el objetivo de este artículo, de ahí que se pretenda recorrer su devenir desde diferentes posturas, hasta llegar a un posicionamiento particular.

Enmarcarlo dentro de un único momento histórico sería incorrecto. Se puede afirmar que sus primeras formas de expresión se encuentran en la tercera generación. Todo esto corresponde con lo expresado por Pérez Luño, cuando afirma que ésta "ha contribuido a redimensionar la nueva imagen del hombre en cuanto sujeto de derechos. Las nuevas condiciones de ejercicio de los derechos humanos han determinado una nueva forma de ser ciudadano en el Estado de derecho de las sociedades tecnológicas". ${ }^{3}$

Sin embargo, la llegada del siglo xxi marcó la identificación de una sociedad donde todo se vinculaba con las tecnologías de la información y las comunicaciones. Por ello, a nuestro criterio, se puede ubicar a partir de este momento una cuarta generación; si se tiene en cuenta la ocurrencia de nuevos fenómenos de agresión a los derechos, dado el desarrollo tecnológico alcanzado o la incidencia de este en todo el entramado social. De ahí la necesidad de contar con herramientas que permitan a las personas reivindicar su derecho a determinar cómo se le dará tratamiento a su información personal.

En esta etapa ya se considera como un derecho autónomo e independiente y, por tanto, se deja de apreciar como parte del derecho a la intimidad o como autodeterminación informativa o libertad informática. El camino transitado ha permitido que se originen iniciativas legislativas y jurisprudenciales que se erigen en fundamento de lo planteado y que más adelante se abordarán.

\section{UN ACERCAMIENTO HISTÓRICO-DOCTRINAL}

El tratamiento, recolección y almacenamiento de informaciones, que antes sólo podían formar parte de la vida íntima de cada ser humano, fue variando paulatinamente el entorno y estructura del derecho a la intimidad. Este último constituye un antecedente importante, si no esencial, de lo que hoy ya se considera como el derecho a la protección de datos de carácter personal y, por tanto, como un derecho fundamental autónomo e independiente. En tal sentido, cualquier análisis debe iniciarse desde sus orígenes y algunos de sus elementos caracterizadores.

El primer enunciado sobre el derecho a la intimidad se encuentra en 1890, en la obra The Right of Privacy ${ }^{4}$ de Samuel D. Warren y Louis B. Brandies, en los Estados Unidos de Norteamérica. Este hecho fue signado por la lucha contra

\footnotetext{
${ }^{3}$ Pérez Luño, Antonio Enrique, La tercera generación de derechos humanos, Navarra, Madrid, Aranzadi, 2006, p. 35.

${ }^{4}$ Véase Elbridge Adams, L., "The Right to Privacy and its Relation to the Law of Libel", American Law Review, enero-febrero, 1905, pp. 37-58.
} 
la interferencia y las intromisiones de la prensa en la vida familiar; con ello se pretendió, además, dejar a salvo a la burguesía de las críticas de la prensa. ${ }^{5}$

En torno a la conceptualización de este derecho, se pueden encontrar diversidad de criterios y autores; sin embargo, tomaremos como referencia aquellos donde se aprecian, a criterio de esta autora, aspectos más distintivos conforme al objeto del presente artículo.

Matilde Zavala de González define el derecho a la intimidad como un derecho que protege la reserva espiritual de la vida privada del hombre, asegurando su libre desenvolvimiento en lo personal, en sus expresiones y en sus afectos. ${ }^{6}$ Nótese cómo el término utilizado es el de "vida privada", aspecto también diverso y polémico en la doctrina, no sólo en el orden terminológico ${ }^{7}$ sino por su contenido.

Yanelis Delgado Triana ${ }^{8}$ considera que protege al individuo frente a las injerencias, intromisiones, vistas, escuchas, publicaciones, captaciones de datos personales, así como el empleo y comunicación, que de algún modo apropien, vulneren o invadan elementos o circunstancias de la dimensión privada del ser humano o de las relaciones inherentes a la estructura y el vivir de la familia.

En esta última se incluye, en el ámbito de salvaguardia del derecho a la intimidad, la protección de los datos de carácter personal. Ello resulta desacertado si se tiene en cuenta que puede transgredirse desde el primero, pero no siempre; por tanto, desde la perspectiva de la citada autora, se expresarían situaciones que quedarían desprovistas de defensa efectiva en el marco del derecho a la intimidad.

Con semejante sentido, Ana María Álvarez Tabío plantea que este derecho amplía su campo para conservar los intereses personales de los individuos con el propósito de resguardarlos de las intromisiones del poder público. A su criterio, el derecho a la intimidad debe reaccionar ante la acumulación de datos destinados al control de los individuos con fines discriminatorios y resguardar el espacio privado del conocimiento ajeno y de la difusión pública de datos reales o supuestos que afecten la esfera íntima de los individuos, e incluye la problemática que genera la paulatina

\footnotetext{
${ }^{5}$ Álvarez Tabío, Ana María (2008), "Los derechos al honor, la intimidad personal y familiar y la propia imagen como límites a las libertades de información y expresión”, Cuba, 2008, pp. 49-51. [Consulta: 19 de agosto, 2012]. Disponible en: http:// www.uh.edu.cu

${ }^{6}$ Zavala de González, Matilde, Derecho a la intimidad, Buenos Aires, Abeledo-Perrot, 1982, p. 87.

${ }^{7}$ La palabra intimidad tiene su origen en el término latino intimus. No sólo se utiliza en países de habla hispana. En el idioma inglés, la palabra intimity suele emplearse para denominar las relaciones sexuales ilícitas; por ello declaran el derecho en cuestión como derecho a la vida privada. El significado de intimidad para la lengua española es en alusión a lo íntimo, secreto, recóndito, profundo, propio. Por tanto, es la zona espiritual íntima y reservada de una persona o de un grupo, especialmente de una familia. Véase Diccionario de la Lengua española, Real Academia española, Vigésima Primera Edición, Madrid, 1992, p. 835.

${ }^{8}$ Delgado Triana, Yanelis, "Protección en el ordenamiento jurídico cubano de los derechos inherentes a la personalidad en la esfera moral", Cuba, 2007, p. 41. [Consulta: 19 de agosto, 2012]. Disponible en: http:// www. uh.edu.cu
} 
acumulación y uso inadecuado de los datos personales. Sin embargo, en este análisis, al igual que en el realizado por Delgado Triana, se confunden los contenidos esenciales de ambos derechos.

Las valoraciones examinadas hasta aquí ubican al derecho a la intimidad con un contenido mucho más amplio, sin delimitarse la existencia aún de un nuevo derecho. Sin embargo, resulta esencial tomar en consideración otras formas que dan continuidad al presente estudio.

La llegada de la década de los setenta del siglo xx marcó, en contextos como el alemán o el italiano, la aparición de una nueva denominación: autodeterminación informativa o libertad informática. En dicho momento se consideró necesario asegurar el ámbito de los ficheros automatizados de datos personales. En Alemania, ${ }^{9}$ por ejemplo, primero se aseveró como un derecho a la personalidad hasta afirmarse como derecho fundamental.

Frosini ${ }^{10}$ es uno de los autores italianos que se afilia a la posición de libertad informática. La califica como derecho perteneciente a la personalidad moral; además la define como el derecho a disponer de los propios datos personales, es decir, controlar la veracidad o exactitud, impedir difundir los datos sensibles o reservados y verificar la utilización para el fin autorizado.

Por su parte, Pérez Luño ${ }^{11}$ lo denomina como libertad informática, propio de la tercera generación, que tiene por finalidad asegurar la facultad de las personas de conocer y acceder a las informaciones archivadas en bancos de datos que les conciernen. Todo ello implica la posibilidad de corregir o cancelar los datos inexactos o indebidamente procesados, y disponer sobre su transmisión.

Concederle esta nueva denominación muestra cómo hasta este periodo existe coincidencia entre los autores en que este derecho responde a todo un proceso evolutivo del derecho a la intimidad de las personas. Por ello, lo reconocen como parte de éste o con una nueva denominación, donde se comienza a asociar la protección de la intimidad frente a la informática, sin llegar aún a definirlo con autonomía.

Adoptar esta postura significa afirmar que con este derecho se asegura al titular de los datos personales la posibilidad de controlar quiénes serán destinatarios de estos y qué uso le darán; para lo cual se debe contar con los derechos de acceso, rectificación y cancelación. Esto conduce, además, a la defensa de principios rectores, como el de dignidad humana.

\footnotetext{
${ }^{9}$ Adquiere rango constitucional a partir de la sentencia del 15 de diciembre de 1983, del Tribunal Constitucional Federal alemán, donde se presenta recurso contra la ley de 25 de marzo de 1982 sobre el censo demográfico, al excederse en la solicitud de información a los ciudadanos.

${ }^{10}$ Francesco Riccobono, Giuffré, "Il nuovo diritto del cittadino", En Nuovi diritti dell'etá tecnológica, Milano, Italia, 1991, p. 6.

${ }^{11}$ Pérez Luño, Antonio Enrique, "Los derechos humanos en la sociedad tecnológica”, en Comisión Andina de Juristas, Perú, 1994, p. 12.
} 


\section{VALORACIONES DESDE UN ÁmbitO NORMATIVO; PARTICULARIDADES EN LATINOAMÉRICA}

El desarrollo de la normativa, tanto en la esfera constitucional como legislativa, manifiesta en Europa, primero, y en Latinoamérica, años más tarde, son evidencia palpable del proceso evolutivo anteriormente señalado. Por ello consideramos oportuno acotar algunos rasgos caracterizadores como fundamento a lo analizado conceptualmente. Tomaremos como punto de análisis algunas normas europeas y latinoamericanas.

El primer antecedente que da origen a la regulación en el ámbito constitucional, del derecho a controlar la información contenido en sistemas de información, se encuentra en la Constitución alemana de Weimar de 1919, específicamente en su artículo 129, párrafos tercero y cuarto, donde se precisa:

Todo funcionario debe tener un recurso contra la decisión disciplinaria que le afecte y la posibilidad de un procedimiento de revisión. Los hechos que le son desfavorables no deben ser anotados en su expediente personal sino después de haberle dado ocasión de justificarse respecto a ellos. El funcionario tiene derecho a examinar su expediente personal. ${ }^{12}$

La Comisión Consultiva, que como parte del Consejo de Europa se creó en 1967, permitió profundizar en el estudio de las problemáticas ocasionadas por el avance de las tecnologías de la información, con énfasis en relación a la vulneración del derecho a la intimidad. ${ }^{13}$ Esto permitió que se aprobaran las resoluciones sobre protección de la intimidad frente a los bancos electrónicos de datos en el sector privado (el 26 de septiembre de 1973) y público (20 de septiembre de 1974). En ambos casos, constituyen los primeros textos supranacionales donde se recogen modelos de conducta para los Estados sobre la protección de datos.

A partir de 1970, se aprecia un desarrollo normativo del derecho a la protección de datos personales, fundamentalmente en Europa. Es válido destacar, por ejemplo, cómo el 7 de octubre de 1970 se promulga la Ley de Hesse en Alemania, considerada pionera en este sentido. En Suecia, la primera ley de protección de datos personales se promulgó en 1973. El objetivo de ambos cuerpos legales fue garantizar los derechos individuales y establecer límites al empleo de la informática.

\footnotetext{
${ }^{12}$ Texto en castellano según la traducción realizada en la obra Las nuevas constituciones del mundo, en 1931. ${ }^{13}$ Este derecho ya había sido establecido por la Declaración Universal de los Derechos Humanos, en el artículo 12, cuando dispone: "Nadie será objeto de injerencias arbitrarias de su vida privada, su familia, su domicilio o su correspondencia, ni de ataques a su honra o a su reputación. Toda persona tiene derecho a la protección de la ley contra tales injerencias o ataques". El Pacto Internacional de Derecho Civiles y Políticos de 1966, en su artículo 17, lo regula prácticamente de la misma forma.
} 
La constitución de Portugal de 1976, en su artículo 35, y la española de 1978, en su artículo 18.4, reconocen el citado derecho. ${ }^{14}$ En el orden de las regulaciones específicas, Francia aprobó, en 1978, la Ley de Informática, Ficheros y Libertades, ${ }^{15}$ relativa a la protección de las personas físicas en relación con el tratamiento de datos de carácter personal. Estas regulaciones se erigen como las primeras muestras donde se declara este derecho y han servido, hasta la actualidad, como punto de referencia para su reconocimiento en otras naciones.

Por su parte, Austria y Luxemburgo, en la propia década de los setenta, dictan disposiciones normativas donde se regulaba el derecho de acceso de las personas a las informaciones que les conciernen, con especial atención a la calidad de los datos y no al hardware que los memoriza. Para ello, se utilizaban cláusulas específicas de protección de la información considerada sensible, debido a su directa incidencia sobre la vida privada o sobre el ejercicio de las libertades.

Todas estas resultan muestras palpables de cómo se ubicaba a este derecho como apéndice del derecho a la intimidad, y se asocia con los primeros momentos del desarrollo de la informática.

A través del Convenio 108 del Consejo de Europa, en 1981, se establecen los principios y derechos que cualquier legislación estatal debía recoger a la hora de proteger los datos de carácter personal. Hasta este momento, la perspectiva era solucionar los problemas que se suscitaban entre el uso de la informática y la intimidad de los sujetos.

La década de los noventa del siglo xx marca el establecimiento de nuevos elementos distintivos. En tal sentido, la Directiva 95/46/CE del Parlamento y Consejo Europeo, del 24 de octubre de 1995, precisa que se garantice la libre circulación de los datos de carácter personal, dado el valor económico que los mismos tienen en las transacciones comerciales.

Con la llegada del siglo Xxi se inicia una nueva etapa en la que se comienza a considerar este derecho como fundamental, autónomo e independiente. La Carta de Derechos Fundamentales de la Unión Europea (Declaración de Niza), de 2000, así lo reconoce en su artículo 8:

1. Toda persona tiene derecho a la protección de los datos de carácter personal que le conciernan. 2. Estos datos se tratarán de modo leal, para fines concretos y sobre la base del consentimiento de la persona

\footnotetext{
${ }_{14}$ Todos ampliados por la Ley Orgánica 15/1999 de Protección de datos de carácter personal (LOPD), del 13 de diciembre de 1999, la cual tuvo como antecedente la Ley Orgánica de Regulación del Tratamiento Automatizado de los datos de carácter personal (LORTAD) de 1992. Sobre esta última y para profundizar sobre el contenido y alcance, véase Davara Rodríguez, Miguel Ángel, "La ley española de protección de datos (LORTAD): ¿una limitación al uso de la informática para garantizar la intimidad?”, Actualidad Furídica, núm. 76-12, Aranzadi, 1992.

${ }^{15}$ Fue modificada por la Ley No. 2004-801, del 6 de agosto de 2004.
} 
afectada o en virtud de otro fundamento legítimo previsto por la ley. Toda persona tiene derecho a acceder a los datos recogidos que la conciernan y a su rectificación. 3. El respeto de estas normas quedará sujeto al control de una autoridad independiente.

La jurisprudencia ha jugado también su papel en la definición del derecho que nos ocupa. Es válido citar sentencias representativas como la del 16 de febrero ${ }^{16}$ y del 4 de mayo ${ }^{17}$ del año 2000, del Tribunal Europeo de Derechos Humanos. En ambas se aboga por la interpretación amplia del derecho a la vida privada y se precisaba que este comprende, además, la posibilidad de establecer y desarrollar relaciones con otros seres humanos, para lo cual se hizo referencia al concepto de dato personal.

Otras sentencias de vital importancia en la declaración de su autonomía resultan ser las 290 y 292 del 30 de noviembre de 2000, del Tribunal Constitucional español. Específicamente, la segunda de ellas consolida la evolución jurisprudencial constitucional que lo ha configurado desde el reconocimiento del derecho a la intimidad, pasando por el denominado derecho a la autodeterminación informática, y reconoce que este derecho fundamental se deriva directamente de la Constitución y por tanto es un derecho independiente de cualquier otro. ${ }^{18}$

Justamente, el fundamento de esta sentencia constituye uno de los más claros, pues enuncia que el contenido específico:

consiste en un poder de disposición y de control sobre los datos personales que faculta a la persona para decidir cuáles de esos datos proporciona a un tercero, sea el Estado o un particular, o cuáles puede este tercero recabar, y que también permite al individuo saber quién posee esos datos personales y para qué, pudiendo oponerse a esa posesión o uso. Estos poderes de disposición y control sobre los datos se concretan jurídicamente en la facultad de consentir la recogida, la obtención y el acceso a los datos personales, su posterior almacenamiento y tratamiento, así como su uso o usos posibles, por un tercero, sea el Estado o un particular.

En Latinoamérica se reconoce la autonomía y rango de derecho fundamental a partir de la Declaración de Santa Cruz de la Sierra, en noviembre de 2003. En este continente, la evolución normativa del derecho se inició con la incorporación en los textos constitucionales, para lo cual se tomaron, en lo

\footnotetext{
${ }^{16}$ Conocida como Sentencia de Amann contra Suiza.

${ }^{17}$ Conocida como Sentencia de Rotura contra Rumanía.

${ }^{18}$ Son válidas, además, las sentencias 110/84, 143/94, 94/98, 11/98, 144/99 y 202/99, todas del Tribunal Constitucional Español.
} 
fundamental, referencias de lo regulado en Portugal y España y se enmarcó en la protección de la intimidad frente a la informática. Sin embargo, se pueden apreciar características distintivas a partir de establecer facultades propias, además de definirse reglas específicas para procesos judiciales preexistentes o la creación de procesos judiciales específicos, como por ejemplo el de hábeas data.

El primer país en regular dicho particular en el ámbito constitucional fue Guatemala, en 1985; mientras que en 1987 se alcanza en Nicaragua. Tanto la Constitución de Guatemala en su artículo 31, como la nicaragüense en su artículo 26, inciso 4, establecen el derecho de toda persona de conocer aquellos datos que sobre ella se encuentren archivados en cualquier tipo de registro estatal y la finalidad que con ellos se persigue. Nótese cómo sólo se establece la posibilidad de conocer sobre la información que conste en registros estatales, no así en los privados, aspecto que para el caso de Nicaragua es ampliado con su ley específica, ${ }^{19}$ aprobada varios años después.

Otro elemento que distingue el precepto constitucional guatemalteco es el de añadir la prohibición de registros de filiación política, exceptuando los que son propios de las autoridades electorales y de los partidos políticos, particular que permite asumirlos como datos sensibles, aunque no los declare de esta forma.

Por su parte, la Constitución brasileña de 1988, en su artículo 5, apartado LXXII, más que consagrar el derecho, regula una garantía específica denominada hábeas data. Se erige, de esta forma, como el primer país que regula este tipo de garantía, dirigida a prevenir la actividad lesiva del Estado frente al tratamiento de datos personales,${ }^{20}$ a la cual coloca en el mismo plano que el hábeas corpus y el mandato de seguranza.

Tres años más tarde, la Constitución de Colombia de 1991 asumió la problemática; se evidenció la preocupación por los avances tecnológicos en materia de informática cuando lo regula en su artículo $15 .^{21}$ Sin seguir al diseño brasileño de reconocer como garantía específica el hábeas data, incorpora un derecho específico que vincula con el derecho a la intimidad y al honor. Destaca, a diferencia de los textos guatemalteco y nicaragüense, el hecho de conceder la posibilidad de acceder a la información que conste en registros públicos y privados.

\footnotetext{
${ }^{19}$ Ley No. 787 “De protección de datos personales", aprobada el 21 de marzo de 2012.

${ }^{20}$ Preceptos ampliados con la Ley 9507/1997, aprobada el 12 de noviembre de 1997, "Del derecho de acceso a la información y recurso procesal de hábeas data".

${ }^{21}$ Preceptos desarrollados, años más tarde, por la Ley Estatutaria 1581/2012, "Disposiciones generales para la protección de datos personales", publicada el 18 de octubre de 2012.
} 
En el propio periodo de los años noventa, la Constitución de Paraguay, ${ }^{22}$ en 1992, lo reconocía en su artículo 135; la peruana, ${ }^{23}$ de 1993, en el artículo segundo, apartados 5, 6 y 7; la Argentina, de 1994, ${ }^{24}$ en el 43, y la venezolana, de 1999, en el 28. En esta última, ya se reconoce el derecho en apartado independiente y se enuncian como sujetos titulares a las personales naturales y jurídicas. Se reconoce como una atribución del Defensor del Pueblo interponer acción de hábeas data; sin embargo, hasta la fecha aún es inexistente una ley específica que regule estos particulares..$^{25}$

La constitución de Argentina, por su parte, lo establece como una forma de la acción de amparo. Se continúa, al igual que en el texto constitucional de Colombia, reconociendo la posibilidad de conocer aquellos almacenados y tratados en registros tanto públicos como privados. Mientras, la constitución peruana permanece en la postura de asociarlo como parte del derecho a la intimidad; de ahí que en los tres apartados anteriormente citados, así se muestre, al igual que en el texto de Paraguay y Colombia.

Otros países, a partir de los primeros años del siglo xxi, continúan en el camino del reconocimiento del derecho objeto de estudio. Tal es el caso de la Constitución mexicana, la cual, tras la modificación de 2007, amplía su artículo 6 y mantiene el 16; en ambos casos constituyen los primeros antecedentes en esta materia. El primero de estos artículos reconoce, desde el primer momento de su aparición en la norma constitucional, el derecho a la información; sin embargo, con la reforma de 2007, se particulariza en su apartado segundo lo referente a protección de los datos personales, con la acotación de que se asegurará en los términos que la ley fije. ${ }^{26}$ De lo anterior, se concluye que en este caso no se le concedía de manera clara la connotación de derecho autónomo, sino asociado al derecho a la intimidad y a la información.

\footnotetext{
${ }^{22}$ Con la Ley 1682/2001, del 16 de enero de 2001, se reglamenta la información de carácter privado. Es modificada por la Ley 1969/2002 del 3 de septiembre de 2002.

${ }^{23}$ Estos preceptos constitucionales se ampliaron con la Ley 29733/2011 "Ley de protección de datos personales", aprobada el 3 de julio de 2011, y su decreto reglamentario 003/2013, del 21 de marzo de 2013.

${ }^{24}$ Con la Ley 25326/2000, "Ley de protección de datos personales", aprobada el 4 de octubre del 2000, reformada por la Ley 26343/2008, del 8 de enero de 2008 y su decreto reglamentario 1558/2001, del 29 de noviembre del 2001, se amplían en este país los preceptos constitucionales.

${ }^{25} \mathrm{Al}$ no existir reglamentación de la figura de hábeas data, el Tribunal Supremo de Justicia, en sentencia de fecha 23 de agosto del 2000, amplió las facultades expresamente reconocidas por la Constitución, las cuales se completaron con las consideraciones emitidas en un informe por la Defensoría del Pueblo. En este se reconoce al hábeas data como un derecho y como una acción. Sin embargo, con la sentencia del 14 de marzo del 2001, de la Sala Constitucional del Tribunal Supremo de Justicia, se adoptó una postura restrictiva, al declarar que no era posible lograr mediante un amparo los objetivos del artículo 28 de la Ley Fundamental. De lo anterior se desprende que en Venezuela, no sólo por no existir norma específica, sino por la inadecuada aplicación, se ha desnaturalizado el precepto constitucional al punto que resulta prácticamente inoperante.

${ }^{26}$ Para lo cual ya existía la Ley Federal de Transparencia y Acceso a la Información Pública Gubernamental, del 30 de abril del 2002, y años más tarde la Ley Federal de Protección de Datos Personales en Posesión de los Particulares, aprobada el 27 de abril del 2010.
} 
Por su parte, la Constitución boliviana de $2009^{27}$ mantuvo la línea de regular este particular como una garantía a la cual denominó acción de protección de privacidad y no como recurso de hábeas data, como lo había hecho en 2002. Sin embargo, resulta inexistente la conformación de un procedimiento específico, sino que el propio precepto constitucional indica que será a través del establecido para la acción de amparo, lo cual puede llevar a plantear si existe una verdadera garantía específica o es simplemente una forma de ejercer la acción de amparo. Además, se sostiene el planteamiento de asociarlo como una forma del derecho a la intimidad.

Un punto importante en este análisis lo revela la normativa uruguaya, si lo preceptuado constitucionalmente tiene, a nuestro criterio, un perfil genérico. ${ }^{28}$ El tratamiento que se logra a partir de su Ley 18.331, de agosto de 2008, ${ }^{29}$ con el consiguiente Decreto Reglamentario No. 414/2009, consagra la protección de datos de carácter personal de forma más acabada, como un derecho inherente a la personalidad humana, además de establecer principios generales, definiciones y otros aspectos vitales para el correcto ejercicio del mismo.

En consecuencia, merece declarar algunos elementos caracterizadores que, en sentido general, pudieran enunciarse conforme al tratamiento normativo de los países latinoamericanos objeto de análisis, sin que lleguen a ser coincidentes en todos los casos:

- Formas diversas de denominar el derecho, en ocasiones asociado al derecho a la intimidad, en otras asociado al derecho a la autodeterminación informativa y en los últimos años asociado al derecho a la información

- Regulación del derecho de forma directa o como una garantía

- Imposibilidad de acceder a los datos de carácter personal que consten en registros privados, al declararse sólo los estatales o públicos

- Falta de reconocimiento de los derechos de rectificación, modificación o cancelación

- Ausencia de una garantía específica con el consiguiente procedimiento

- Declaración del hábeas data sin una forma común, en tanto se declara como una acción o como un recurso

- Los sujetos titulares pueden ser tanto personales y naturales como jurídicos

- Apego a la regulación europea sin rasgos que muestren las realidades de cada una de sus sociedades

\footnotetext{
${ }^{27}$ Reconoció este particular desde 1995 en el artículo 23 y lo mantuvo con la reforma de 2002 y 2004.

${ }^{28}$ En el artículo 72 del texto constitucional uruguayo se establece que "La enumeración de derechos, deberes y garantías hecha por la Constitución no excluye los otros que son inherentes a la personalidad humana o se derivan de la forma republicana de gobierno".

${ }^{29}$ Modificada por la Ley 18719/2010 del 27 de diciembre del 2010.
} 


\section{CONCLUSIONES}

Todo lo analizado revela que, desde la década de los setenta del siglo xx, se supera de forma progresiva el concepto restringido del derecho a la intimidad, que, aplicado a los avances tecnológicos, da paso a la denominación del derecho a la autodeterminación informativa o libertad informática. Años más tarde, emerge, con independencia y autonomía, un nuevo derecho fundamental, denominado derecho a la protección de datos personales.

El desarrollo normativo actual de este último, además, muestra que con él se persigue garantizar el control de los individuos respecto a sus datos personales, así como el uso y destino de estos a fin de evitar su intercambio fraudulento y dañoso.

Para los países latinoamericanos, en el proceso de reconocimiento de este derecho, generalmente la prioridad del Estado se centró en reconocer, dentro de las limitaciones económicas presentes en muchos de ellos, el derecho a prepararse sobre la base de las nuevas tecnologías y el acceso apropiado a las líneas. Es por ello que quedó para etapas posteriores; específicamente, a partir de finales de la década de los ochenta del siglo Xx.

Progresivamente, en esta área geográfica, se fue reconociendo como autónomo e independiente, con base constitucional, fundamentalmente, o mediante legislaciones específicas. Sin embargo, es vital tomar en consideración las tradiciones jurídicas y las realidades políticas, económicas y sociales presentes en estas sociedades, pues hasta el momento se refleja, mayormente, la línea normativa de Europa.

La multiplicación que se expresa debe continuar extendiéndose a otras latitudes, como es el caso de Cuba, donde si bien se han dado pasos precisos en cuanto a informatizar toda la sociedad y se trazan estrategias atinadas en este sentido, aún es inexistente la regulación de este derecho.

Llevar a vías de hecho la prioridad que exige para este continente contar con normas y principios bases para la protección de la información personal debe constar en las agendas políticas de nuestros países, esencialmente de aquellos organismos creados y envestidos para cumplimentar dichas funciones.

Continuar adentrándose en el estudio de este derecho implica indagar no sólo en los niveles de conocimiento que la ciudadanía tiene en cuanto al valor de sus datos personales, sino en el peligro que supone la irresponsabilidad conciudadana en el tratamiento y almacenamiento de los mismos. 


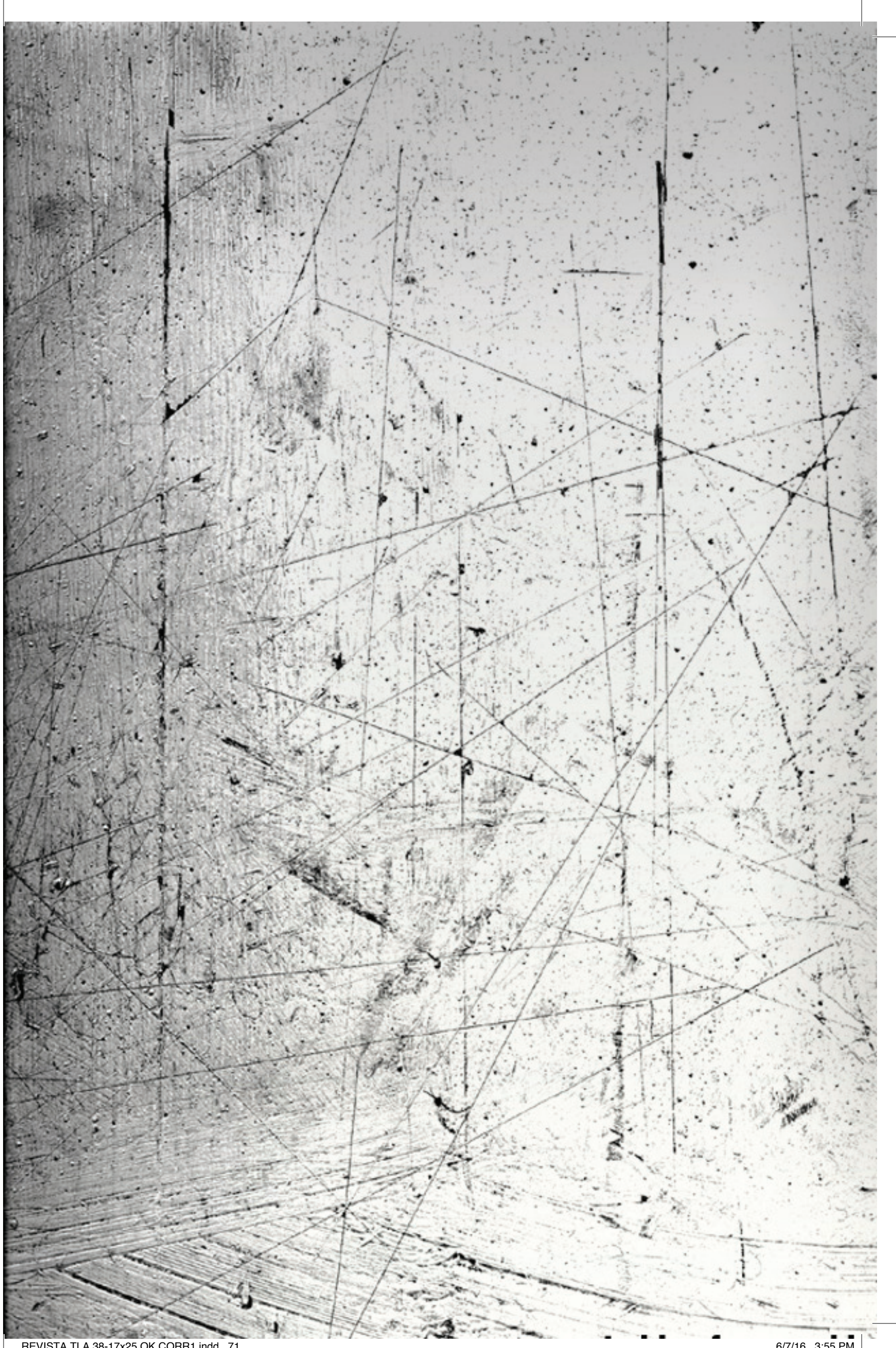

